\title{
Antibiotic Use and Treatment Outcomes among Children with Community-Acquired Pneumonia Admitted to a Tertiary Care Public Hospital in Nepal
}

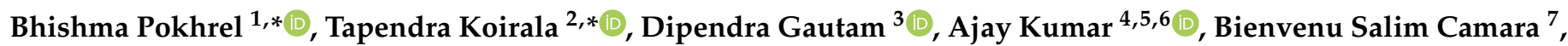 \\ Saw Saw ${ }^{8}$, Sunil Kumar Daha ${ }^{1}$, Sunaina Gurung ${ }^{1}$, Animesh Khulal ${ }^{1}$, Sonu Kumar Yadav ${ }^{1}$, Pinky Baral ${ }^{9}$, \\ Meeru Gurung ${ }^{1}$ D and Shrijana Shrestha ${ }^{1}$
}

1 Patan Hospital, Patan Academy of Health Sciences, Lalitpur 44700, Nepal; sunilkumardaha@pahs.edu.np (S.K.D.); sunaina.the.g@gmail.com (S.G.); anmshkbassnet@gmail.com (A.K.); sonukumaryadav@pahs.edu.np (S.K.Y.); drmeeru@hotmail.com (M.G.); shrijanashrestha@pahs.edu.np (S.S.)

2 Department of Health Services, Ministry of Health and Population, Kathmandu 44600, Nepal

3 World Health Emergencies Program, WHO Country Office, Kathmandu 44600, Nepal; gtmdipen@gmail.com

4 International Union against Tuberculosis and Lung Disease, South-East Asia Office, New Delhi 110016, India; AKumar@theunion.org

5 International Union against Tuberculosis and Lung Disease, 75006 Paris, France

check for updates

Citation: Pokhrel, B.; Koirala, T.; Gautam, D.; Kumar, A.; Camara, B.S.; Saw, S.; Daha, S.K.; Gurung, S.; Khulal, A.; Yadav, S.K.; et al. Antibiotic Use and Treatment Outcomes among Children with Community-Acquired Pneumonia Admitted to a Tertiary Care Public Hospital in Nepal. Trop. Med. Infect. Dis. 2021, 6, 55. https://doi.org/ 10.3390 /tropicalmed6020055

Academic Editors: Olga Perovic,

Tom Decroo and Chakaya

Muhwa Jeremiah

Received: 2 March 2021

Accepted: 24 March 2021

Published: 20 April 2021

Publisher's Note: MDPI stays neutral with regard to jurisdictional claims in published maps and institutional affiliations.

Copyright: (c) 2021 by the authors. Licensee MDPI, Basel, Switzerland. This article is an open access article distributed under the terms and conditions of the Creative Commons Attribution (CC BY) license (https:// creativecommons.org/licenses/by/ $4.0 /)$.
6 Yenepoya Medical College, Yenepoya, Mangaluru 575018, India

7 Central National de Formation et de Recherche en Santé Rurale de Maferinyah, Forécariah 4090, Guinea; bscamara@maferinyah.org

8 Department of Medical Research, Ministry of Health and Sports, Yangon 05081, Myanmar; sawsaw@mohs.gov.mm

9 Modern Technical College, Sanepa, Lalitpur 44700, Nepal; baralpinky@gmail.com

* Correspondence: bhishmapokhrel@pahs.edu.np (B.P.); tapendrakoirala@gmail.com (T.K.); Tel.: +977-15522278/5522266 (B.P.); +977-9863328938 (T.K.)

\begin{abstract}
In the era of growing antimicrobial resistance, there is a concern about the effectiveness of first-line antibiotics such as ampicillin in children hospitalized with community-acquired pneumonia. In this study, we describe antibiotic use and treatment outcomes among under-five children with community-acquired pneumonia admitted to a tertiary care public hospital in Nepal from 2017 to 2019. In this cross-sectional study involving secondary analysis of hospital data, there were 659 patients and $30 \%$ of them had a history of prehospital antibiotic use. Irrespective of prehospital antibiotic use, ampicillin monotherapy (70\%) was the most common first-line treatment provided during hospitalization followed by ceftriaxone monotherapy (12\%). The remaining children (18\%) were treated with various other antibiotics alone or in combination as first-line treatment. Broadspectrum antibiotics such as linezolid, vancomycin, and meropenem were used in less than $1 \%$ of patients. Overall, $66(10 \%)$ children were required to switch to second-line treatment and only $7(1 \%)$ children were required to switch to third-line treatment. Almost all (99\%) children recovered without any sequelae. This study highlights the effectiveness of ampicillin monotherapy in the treatment of community-acquired pneumonia in hospitalized children in a non-intensive care unit setting.
\end{abstract}

Keywords: community-acquired pneumonia; CAP; antibiotic use; treatment outcome; operational research; SORT IT

\section{Introduction}

Pneumonia remains the most important cause of child mortality beyond the neonatal period globally [1], and also the most important cause of under-five mortality in south-east Asia [2]. It was estimated that around 65,000 children died of pneumonia in 2016 globally with more than $85 \%$ occurring in south-east Asian countries. The annual incidence of pneumonia among under-five children in south-east Asia is estimated to be 0.36 episodes per child [3]. In Nepal, pneumonia accounted for $10 \%$ of all childhood acute respiratory 
infections (ARI) in 2017 with an annual incidence of 66 cases per 1000 under-five children [4]. Previous studies from tertiary care settings in Nepal show that most of the pneumonia in under-five children were viral in origin and those with bacterial etiology were sensitive to the penicillin group of antibiotics $[5,6]$.

A clinical case management approach formulated by the World Health Organization (WHO) is in place to reduce pneumonia-related deaths and improve access to life-saving antibiotics for children with community-acquired pneumonia (CAP), and to prevent unnecessary antibiotic use and hospitalizations in those who can be safely managed as outpatients [7]. WHO recommends facility-based parenteral ampicillin and gentamicin as the empirical therapy to treat all severe pneumonia whereas home-based oral amoxicillin for all non-severe pneumonia on an outpatient basis is recommended. However, large variation is seen in antibiotic use within and across countries, with inappropriate use of antibiotics common in low- and middle-income countries. Increased utilization of antibiotics without prescription has been reported in developing countries, with $44 \%$ to $97 \%$ of patients receiving inappropriate antibiotics [8-10]. The irrational use and overuse of antibiotics may lead to the emergence of antimicrobial resistance (AMR), especially in resource-limited countries, due to unregulated distribution of antibiotics in the population, inappropriate health provider practices such as the wrong prescription of antibiotics, self-medication, and incomplete intake of prescribed antibiotic doses by the patients [11].

With the growing threat of AMR in south-east Asia [12], understanding the pattern of antibiotics used to treat common pediatric illnesses like CAP is crucial. There is limited information on antibiotic regimens used to treat CAP in tertiary level hospitals in Nepal. Hence, we conducted an operational research study, among the most vulnerable age group (2-59 months) admitted with CAP to the Patan hospital in Nepal, with the following objectives: i) to describe the socio-demographic and clinical characteristics, ii) to determine the yield of blood culture, and iii) to describe the patterns of antibiotic regimens used for treatment during hospitalization and treatment outcomes.

\section{Materials and Methods}

\subsection{Study Design}

This was a cross-sectional study using routinely collected secondary data from the electronic records and paper-based patient files.

\subsection{Study Setting}

\subsubsection{General Setting}

Nepal is a small landlocked country in south Asia, lying between two large countries, India and China. It is composed of seven federal provinces with a population of 30 million people (as of 2017). About 35\% of the population are children aged less than 15 years [13] and about $40 \%$ live below the poverty line [14]. The health system of Nepal consists of 125 public hospitals and 1822 non-public hospitals. There are three levels of health facilities ranging from local (primary), to provincial (secondary), to central (tertiary). From 2013 to 2018, the per capita government spending on health gradually increased from Nepalese rupees (NPR) 966 to NPR 1819 (USD 9.8 to 17.7) [15].

\subsubsection{Specific Setting}

The study was conducted at the Patan Hospital, a 640-bedded autonomous, not-forprofit, tertiary level public hospital located in the southern part of Lalitpur district of province 3. It is one of the WHO sentinel sites for the surveillance of invasive bacterial diseases, namely pneumonia, sepsis, and meningitis. The hospital has an average of 320,000 outpatient visits and 20,000 admissions annually.

\subsubsection{Protocol Used at the Hospital}

Patan Hospital has a 54-bedded general pediatric ward apart from 12-bedded intensive care units (pediatric ICU and neonatal ICU) and 20-bedded neonatal nurseries. 
Identification of CAP is based on a clinical diagnosis by the pediatrician in any child with a history of cough and/or shortness of breath, with or without fever, and relevant findings on systemic examination aided by radiological evidence as defined by the Infectious Diseases Society of America (IDSA) [14]. For the treatment of CAP, various factors like the severity of illness, prior antibiotic use, underlying comorbidities, nutritional status, and radiological findings on chest radiograph play an important role in helping the pediatrician to decide which antibiotic to be used. The antibiotic used in the first $48 \mathrm{~h}$ is considered the first-line antibiotic. Ampicillin with or without amikacin, cloxacillin, and azithromycin are commonly used as the first-line antibiotics. If there is no clinical improvement by $48 \mathrm{~h}$ after starting first-line treatment or if there is rapid deterioration within $48 \mathrm{~h}$, the antibiotic is changed (second-line treatment). Antibiotics like ceftriaxone, cefotaxime, chloramphenicol, vancomycin, and linezolid are often used as second-line antibiotics. If clinical improvement does not occur within $48 \mathrm{~h}$ of starting second-line antibiotics, the treating pediatrician considers upgrading the antibiotics to the third-line antibiotics. Meropenem and colistin are considered the third-line antibiotics. However, antibiotics that are considered as the second- or third-line may be used as first-line or second-line, especially when the child has a history of prehospital antibiotic use and clinically severe. Before starting antibiotic treatment, a blood sample is collected from each patient for culture and drug susceptibility test as a part of WHO invasive bacterial disease surveillance, which takes at least $72 \mathrm{~h}$ before results are available. If any bacterial pathogen is identified from the blood culture, the antibiotic is modified based on the culture and sensitivity pattern.

\subsection{Study Population}

We included all children aged 2-59 months with a diagnosis of CAP admitted to the pediatric ward of the Patan Hospital from 1 January 2017 to 31 December 2019. For this study, we defined CAP as "an acute infection of the pulmonary parenchyma that is associated with some symptoms of acute infection, accompanied by the presence of an acute infiltrate on a chest radiograph or auscultatory findings consistent with pneumonia in a patient not hospitalized or residing in a long-term care facility for more than 14 days before the onset of symptoms" as per IDSA guidelines [16]. The manifestations of acute infection include fever, cough, age-specific tachypnea, and lower chest wall indrawing; the auscultatory findings include abnormal breath sounds, wheezes, or crackles; and the radiographic evidence includes consolidation, other infiltrate, or pleural effusion.

The exclusion criteria included:

1. Children who had complicated pneumonia (as defined by the presence of significant effusion, empyema, necrotizing pneumonia, pneumothorax, severe or impending respiratory failure, and/or signs and symptoms of sepsis or shock) at the time of admission.

2. Persistent (chronic) pneumonia syndromes (with symptoms for $>2$ weeks).

3. History suggestive of aspiration pneumonia or recurrent pneumonia.

4. Pneumonia associated with chronic medical problems such as immunodeficiency such as diabetes mellitus, chronic kidney disease, bronchial asthma, lung malignancy, known HIV infection.

5. Invasive mechanical ventilation within 14 days before the current hospital admission.

6. Children who have taken antibiotics for a respiratory infection for more than 7 days before the hospital admission.

7. History of cystic fibrosis, post-obstructive pneumonia, or active tuberculosis.

\subsection{Data Variables and Sources}

Data were collected between March and December 2020. Data variables included age, sex, date of hospital admission, chest radiograph findings, nutritional status, the severity of pneumonia (defined by the presence of any of the danger signs such as convulsions, lethargy or altered sensorium, persistent vomiting, poor oral intake, and cyanosis as per the WHO definition) [7], history of prehospital antibiotic use, blood culture results, antibiotics 
used in first-line, second-line, and third-line treatment, and treatment outcomes. Data sources included the patient files (both electronic and paper-based) and the laboratory register of the microbiology department.

\subsection{Analysis and Statistics}

The data collected from the patient charts were double entered and validated using EpiData version 3.1 (EpiData Association, Odense, Denmark). This was merged with the electronic data using a case identification number and a master dataset prepared for analysis. Data were reviewed for inconsistencies and cleaned before analysis. Data were analyzed using EpiData Analysis software (version 2.2.2.187). Descriptive analysis was conducted and presented as frequencies and proportions for categorical data.

\section{Results}

\subsection{Sociodemographic and Clinical Characteristics}

Overall, 659 children were admitted with CAP; $57 \%$ were females and $46 \%$ were infants aged less than 12 months. Of these, only $26 \%$ met the WHO criteria for severe pneumonia whereas 39\% did not meet the WHO criteria for pneumonia. The majority of children (66\%) had normal chest radiography findings. Among children with WHO-defined severe CAP, only $10 \%$ had positive findings in their chest radiography. Prehospital antibiotic use was seen in $30 \%$ of children (Table 1). Wheezing was seen in $214(32 \%)$ children.

Table 1. Sociodemographic and clinical characteristics of children (aged 2-59 months) with community-acquired pneumonia admitted to Patan hospital, Nepal during 2017-2019 (n=659).

\begin{tabular}{|c|c|c|}
\hline Characteristic & Number & Percentage \\
\hline \multicolumn{3}{|l|}{ Age (months) } \\
\hline $2-11$ & 306 & $(46.4)$ \\
\hline $12-23$ & 155 & $(23.5)$ \\
\hline $24-35$ & 108 & $(16.4)$ \\
\hline $36-47$ & 61 & $(9.3)$ \\
\hline $48-59$ & 29 & $(4.4)$ \\
\hline \multicolumn{3}{|l|}{ Gender } \\
\hline Female & 376 & $(57.1)$ \\
\hline Male & 283 & $(42.9)$ \\
\hline \multicolumn{3}{|l|}{ Residence } \\
\hline Lalitpur & 488 & $(74.1)$ \\
\hline Bhaktapur & 23 & $(3.5)$ \\
\hline Kathmandu & 80 & $(12.1)$ \\
\hline Others & 68 & $(10.3)$ \\
\hline \multicolumn{3}{|l|}{ History of PCV * vaccination } \\
\hline Received complete immunization as per age & 447 & $(67.8)$ \\
\hline Incomplete immunization as per age & 74 & $(11.2)$ \\
\hline $\begin{array}{l}\text { Immunized but the number of doses not } \\
\text { available }\end{array}$ & 81 & $(12.3)$ \\
\hline No immunization received & 57 & $(8.6)$ \\
\hline \multicolumn{3}{|l|}{ History of Hib vaccination } \\
\hline Received complete immunization as per age & 316 & $(48.0)$ \\
\hline Incomplete immunization as per age & 11 & $(1.7)$ \\
\hline $\begin{array}{l}\text { Immunized but the number of doses not } \\
\text { available }\end{array}$ & 319 & $(48.4)$ \\
\hline No immunization received & 13 & $(2.0)$ \\
\hline \multicolumn{3}{|l|}{ Nutritional status } \\
\hline Normal & 477 & $(72.4)$ \\
\hline Stunting & 78 & $(11.8)$ \\
\hline Wasting & 60 & $(9.1)$ \\
\hline Stunting and wasting & 44 & (6.7) \\
\hline
\end{tabular}


Table 1. Cont.

\begin{tabular}{ccc}
\hline Characteristic & Number & Percentage \\
\hline WHO severity classification & & \\
No pneumonia & 255 & $(38.7)$ \\
Pneumonia & 231 & $(35.1)$ \\
Severe pneumonia & 173 & $(26.3)$ \\
Chest radiology & & \\
Normal & 436 & $(66.2)$ \\
Infiltrates & 139 & $(21.1)$ \\
End-point consolidation & 74 & $(1.2)$ \\
Uninterpretable & 10 & $(30.2)$ \\
Prehospital antibiotic use & & $(58.4)$ \\
Yes & 199 & $(11.4)$ \\
No & 385 & \\
Unknown & 75 & \\
\hline
\end{tabular}

* PCV-Pneumococcal conjugate vaccine; Hib-Hemophilus Influenzae B vaccine.

Most of the children reported a history of immunization using pneumococcal conjugate vaccine (PCV) (91\%) and Hemophilus Influenzae B (Hib) (98\%) vaccines, but the information on the number of doses of vaccine received was missing in $12 \%$ of children for PCV and $48 \%$ of children for Hib vaccine- this precluded assessment of completeness of vaccination appropriate to the age. Overall, the completeness of vaccination was better with PCV compared to the Hib vaccine (68\% versus $48 \%$ ).

\subsection{Blood Culture Results}

Of the 659 patients, $646(98 \%)$ had their blood culture done and only $5(0.8 \%)$ patients were culture-positive for bacterial pathogens (three patients with coagulase-negative Staphylococcus (CoNS), one Streptococcus pneumoniae, and one Streptococcus viridans). CoNS were considered "contaminants" and thus no treatment changes were required. Both Streptococcus pneumoniae and Streptococcus viridans were fully sensitive to the penicillin group of drugs including ampicillin.

\subsection{Antibiotic Use during Hospitalization}

The antibiotic regimens used as first-line treatment after hospital admission is depicted in Table 2. Irrespective of prehospital antibiotic use, ampicillin monotherapy $(69.5 \%)$ followed by ceftriaxone monotherapy $(12.3 \%)$ were the two most common first-line antibiotics regimens used. The remaining children $(18 \%)$ were treated with other antibiotics as firstline treatment and there was a total of 23 different regimens used. Overall, $66(10 \%)$ children were required to switch to second-line antibiotics and only $7(1 \%)$ children were required to switch to third-line antibiotics. The proportion of children who received ampicillin monotherapy and subsequently required to upgrade to second-line and third-line were $11.6 \%$ and $0.4 \%$, respectively, while that for the ceftriaxone monotherapy group was $4.9 \%$ and none, respectively (Table 3). Among children with WHO-defined severe pneumonia receiving ampicillin monotherapy, only $4.6 \%$ required second-line treatment. All children (except two who received oral azithromycin) were started on intravenous antibiotics. The overall use of antibiotics is shown in Table 4 which shows that Ampicillin was used in 80\% of the children (either singly or in combination) followed by Ceftriaxone and Cloxacillin. 
Table 2. Patterns of antibiotic treatment received during hospitalization by children (aged 2-59 months) with community-acquired pneumonia admitted to Patan hospital, Nepal during 2017-2019.

\begin{tabular}{|c|c|c|c|c|c|c|c|c|}
\hline \multirow[t]{2}{*}{ Treatment Regimen } & \multicolumn{2}{|c|}{$\begin{array}{c}\text { Prehospital } \\
\text { Antibiotic Use } \\
\text { (199) }\end{array}$} & \multicolumn{2}{|c|}{$\begin{array}{c}\text { No Prehospital } \\
\text { Antibiotic Use } \\
\text { (385) }\end{array}$} & \multicolumn{2}{|c|}{$\begin{array}{l}\text { Unknown } \\
\text { Status } \\
(75)\end{array}$} & \multicolumn{2}{|c|}{$\begin{array}{l}\text { Total } \\
(659)\end{array}$} \\
\hline & $n$ & $(\%)$ & $n$ & $(\%)$ & $n$ & $(\%)$ & $n$ & $(\%)$ \\
\hline AMP & 127 & $(63.8)$ & 282 & $(73.2)$ & 49 & $(65.3)$ & 458 & $(69.5)$ \\
\hline $\mathrm{AMP}+\mathrm{AMK}$ & 13 & $(6.5)$ & 16 & $(4.1)$ & 4 & $(5.3)$ & 33 & $(5.0)$ \\
\hline $\mathrm{AMP}+\mathrm{AZT}$ & 4 & $(2.0)$ & 11 & $(2.9)$ & 1 & $(1.3)$ & 16 & $(2.4)$ \\
\hline $\mathrm{AMP}+\mathrm{CFTR}$ & 0 & $(0.0)$ & 2 & $(0.5)$ & 3 & $(4.0)$ & 5 & $(0.8)$ \\
\hline $\mathrm{AMP}+\mathrm{OFX}$ & 0 & $(0.0)$ & 2 & (0.5) & 0 & $(0.0)$ & 2 & $(0.3)$ \\
\hline $\mathrm{AMP}+\mathrm{CLX}$ & 0 & $(0.0)$ & 8 & (2.1) & 1 & $(1.3)$ & 9 & (1.4) \\
\hline $\mathrm{AMP}+\mathrm{CFTR}+\mathrm{CLP}$ & 1 & (0.5) & 0 & $(0.0)$ & 0 & $(0.0)$ & 1 & $(0.2)$ \\
\hline $\mathrm{AMP}+\mathrm{AMK}+\mathrm{CFTX}$ & 1 & $(0.5)$ & 2 & $(0.5)$ & 1 & $(1.3)$ & 4 & $(0.6)$ \\
\hline CFTR & 36 & (18.1) & 37 & $(9.6)$ & 8 & $(10.7)$ & 81 & (12.3) \\
\hline CFTR + CLX & 5 & $(2.5)$ & 14 & (3.6) & 3 & $(4.0)$ & 22 & (3.3) \\
\hline CFTR + AZT & 2 & $(1.0)$ & 2 & $(0.5)$ & 1 & $(1.3)$ & 5 & $(0.8)$ \\
\hline CFTR + MTRZ & 1 & $(0.5)$ & 0 & $(0.0)$ & 0 & $(0.0)$ & 1 & $(0.2)$ \\
\hline CFTR + CIP & 1 & (0.5) & 0 & $(0.0)$ & 0 & $(0.0)$ & 1 & $(0.2)$ \\
\hline CFTR + CLX + CLP & 0 & $(0.0)$ & 0 & $(0.0)$ & 1 & $(1.3)$ & 1 & $(0.2)$ \\
\hline CLX & 0 & $(0.0)$ & 1 & (0.3) & 0 & $(0.0)$ & 1 & $(0.2)$ \\
\hline CLX + CLP & 0 & $(0.0)$ & 0 & $(0.0)$ & 1 & $(1.3)$ & 1 & $(0.2)$ \\
\hline CLX + MTRZ & 1 & $(0.5)$ & 0 & $(0.0)$ & 0 & $(0.0)$ & 1 & $(0.2)$ \\
\hline CLX + GEN & 0 & $(0.0)$ & 1 & (0.3) & 0 & $(0.0)$ & 1 & $(0.2)$ \\
\hline $\mathrm{AZT}$ & 0 & $(0.0)$ & 2 & $(0.5)$ & 0 & $(0.0)$ & 2 & $(0.3)$ \\
\hline $\mathrm{AZT}+\mathrm{CIP}$ & 1 & (0.5) & 0 & $(0.0)$ & 0 & $(0.0)$ & 1 & $(0.2)$ \\
\hline $\mathrm{AZT}+\mathrm{CLP}+\mathrm{VAN}$ & 1 & (0.5) & 0 & $(0.0)$ & 0 & $(0.0)$ & 1 & $(0.2)$ \\
\hline CLP & 3 & (1.5) & 1 & $(0.3)$ & 1 & $(1.3)$ & 5 & $(0.8)$ \\
\hline MERO & 0 & $(0.0)$ & 0 & $(0.0)$ & 1 & $(1.3)$ & 1 & $(0.2)$ \\
\hline CIP & 0 & $(0.0)$ & 1 & (0.3) & 0 & $(0.0)$ & 1 & $(0.2)$ \\
\hline CFTX & 1 & (0.5) & 0 & $(0.0)$ & 0 & $(0.0)$ & 1 & $(0.2)$ \\
\hline Not recorded & 1 & (0.5) & 3 & (0.8) & 0 & $(0.0)$ & 4 & (0.6) \\
\hline
\end{tabular}

AMP-Ampicillin; AMK-Amikacin; AZT-Azithromycin; CFTR-Ceftriaxone; OFX-Ofloxacin; CLXCloxacillin; CLP-Chloramphenicol; CFTX-Cefotaxime; MTRZ-Metronidazole; CIP-Ciprofloxacin; GENGentamicin; VAN—Vancomycin; MERO-Meropenem.

Table 3. Proportion of children who required switch to second-line and third-line antibiotics among children (aged 2-59 months) with community-acquired pneumonia admitted to Patan hospital, Nepal during 2017-2019.

\begin{tabular}{ccccc}
\hline \multirow{2}{*}{$\begin{array}{c}\text { Treatment Group (Based on } \\
\text { First-Line Treatment) }\end{array}$} & \multicolumn{3}{c}{ Antibiotic Upgraded to } & Total \\
\cline { 2 - 4 } & $\boldsymbol{n} \mathbf{( \% )}$ & $\begin{array}{c}\text { Second-Line } \\
\boldsymbol{n} \mathbf{( \% )}\end{array}$ & $\begin{array}{c}\text { Third-Line } \\
\boldsymbol{n} \mathbf{( \% )}\end{array}$ & $\boldsymbol{n} \mathbf{( \% )}$ \\
\hline Ampicillin alone & $403(88.0)$ & $53(11.6)$ & $2(0.4)$ & $458(100)$ \\
Ceftriaxone alone & $77(95.1)$ & $4(4.9)$ & $0(0.0)$ & $81(100)$ \\
Other regimens & $100(83.3)$ & $15(12.5)$ & $5(4.2)$ & $120(100)$ \\
Total & $580(88.0)$ & $72(10.9)$ & $7(1.1)$ & $659(100)$ \\
\hline
\end{tabular}

\subsection{Treatment Outcomes}

Of 659 children, 651 (99\%) recovered. A total of five children left the hospital against medical advice while on treatment and two were transferred to the intensive care unit. One patient was sent home after $72 \mathrm{~h}$ of hospitalization on oral therapy as per the parent's request. 
Table 4. Overall usage of antibiotics among children (aged 2-59 months) with CAP admitted to Patan hospital, Nepal during 2017-2019 ( $n=659)$.

\begin{tabular}{ccc}
\hline Antibiotic & $\boldsymbol{n}$ & $\mathbf{( \% )}$ \\
\hline Ampicillin & 530 & $(80.4)$ \\
Ceftriaxone & 157 & $(23.8)$ \\
Cloxacillin & 44 & $(6.7)$ \\
Amikacin & 38 & $(5.8)$ \\
Azithromycin & 31 & $(4.7)$ \\
Cloramphenicol & 26 & $(3.9)$ \\
Cefotaxim & 7 & $(1.1)$ \\
Vancomycin & 6 & $(0.9)$ \\
Ofloxacin & 6 & $(0.9)$ \\
Meropenem & 5 & $(0.8)$ \\
Linezolid & 2 & $(0.3)$ \\
Others \# & 9 & $(1.4)$ \\
\hline
\end{tabular}

* Percentages are calculated using a total of 659 children as the denominator. \# Cotrimoxazole, Clindamycin, Durataz (Piperacillin sodium and Tazobactam sodium), Ciprofloxacin, Gentamicin, Metronidazole.

\section{Discussion}

Antimicrobial resistance is a serious public health threat globally. The present study is one of the few from Nepal to carry out a situation analysis on the use of antibiotics to treat CAP in hospitalized children aged 2-59 months. In our study, the majority (70\%) of the children were treated with ampicillin monotherapy and among them, nearly nine in ten children had an uneventful recovery without the need for antibiotic upgrade irrespective of prehospital antibiotic use. The use of ceftriaxone as a first-line antibiotic was low $(12.3 \%)$. There exists significant variation in the use of empirical first-line antibiotics recommended by various guidelines in the treatment of CAP in hospitalized children. WHO's recent recommendations emphasize the parenteral use of ampicillin and gentamicin in the treatment of severe CAP [7], whereas the American Academy of Pediatrics (AAP) recommends ampicillin as the first-line antibiotic for uncomplicated pediatric CAP requiring hospitalization [17].

In our study, nearly seven in ten children who met the WHO criteria for severe pneumonia were also treated with ampicillin monotherapy without any need for upgrade into second- or third-line antibiotics. Only $4.6 \%$ of those children needed an antibiotic upgrade. This finding is in agreement with the recommendation by the AAP for the treatment of uncomplicated pediatric CAP. We did not find any published literature comparing the treatment outcome of ampicillin alone versus ampicillin in combination with gentamicin in the treatment of pneumonia as per WHO classification. However, ampicillin has been compared with various other antibiotics in the treatment of uncomplicated CAP in hospitalized children. One study from Israel found comparable treatment outcomes between the penicillin/ampicillin group and cefuroxime group [18].

There have been several other studies that have shown the use of third-generation cephalosporins as the first-line treatment in pediatric CAP. Such broad-spectrum antibiotics have been shown to contribute to antimicrobial resistance and Clostridium difficile infection [17], and should be used judiciously only where there is a strong suspicion of highly penicillin-resistant Streptococcus pneumoniae or beta-lactamase producing Haemophilus influenzae [19]. Antibiotic use before the hospital admission was seen in $30 \%$ of the children in our study. Other studies from Asian countries like the Philippines (17-53\%), Bhutan $(30.4 \%)$, and Vietnam $(49.1 \%)$ reported similar findings [20-22]. Easy availability and sale of antibiotics over-the-counter could be one of the main reasons for such a high prevalence of prehospital antibiotic use among the study participants, which might contribute to antimicrobial resistance in the future.

The yield of blood culture was extremely low $(0.75 \%)$ in this study. Only two pathogens (Streptococcus pneumoniae and Streptococcus viridians) were isolated and they were fully susceptible to the penicillin group of drugs. Various studies have reported a wide 
range of bacteremia in pediatric CAP (0.8-17.4\%) [23]. Therefore, studies conducted in non-ICU settings have recommended that routine use of blood culture may not be needed for most children hospitalized with CAP [24], as it has been found that viruses are the most common causes of CAP in healthy, immunized children, and bacteria account for $\sim 15 \%$ of the cases of which Streptococcus pneumoniae is the most common pathogen $[25,26]$. The routine immunization against Streptococcus pneumoniae and Haemophilus influenzae type B in Nepal has resulted in a decrease in pediatric CAP secondary to these invasive bacterial infections, from $7.7 \%$ to $4 \%[23,27]$. However, children admitted in ICU settings with complicated pneumonia may benefit from the routine performance of blood cultures [28].

Though all the patients enrolled in our study had their chest radiography performed, only one-third of them had radiological evidence of pneumonia (32.3\%). Among the children with WHO severe pneumonia, the chest radiograph was normal in $61.8 \%$ and only $9.6 \%$ had radiological evidence of pneumonia. In a study in Pakistani children with non-severe pneumonia, the radiological evidence of pneumonia was only found in $14 \%$ of cases as per WHO criteria [29]. A similar finding has been reported from a study done in Ethiopia which showed that $51.6 \%$ of the children diagnosed with severe pneumonia had no radiological evidence of pneumonia [30]. Studies from developed countries like the USA have also revealed similar findings in pediatric CAP [31,32]. Children with normal X-ray and low clinical suspicion of pneumonia can be observed without antibiotic therapy [33].

Our study had some strengths. First, we had a large sample size covering a period of three years. We used data collected routinely in our setting and hence the findings reflect the programmatic realities on the ground. Since the study site is a WHO surveillance center, the quality of the routine data related to pneumonia was good with periodic validation by dedicated and trained staff. Hence, the findings are valid and reliable.

We also had some limitations. First, since the study was conducted in a single tertiary care hospital, findings are not widely generalizable. Second, we might have overestimated the positive outcomes because of our strict exclusion criteria which excluded many children with more severe disease. Third, the information on additional supportive treatments provided (such as oxygen therapy, nebulization with beta- 2 agonists, and others) and the main reason for admission were not captured in the electronic database. Hence, we are unable to report on these variables.

Despite these limitations, there are many implications for policy and practice. First, the majority of children with pneumonia admitted to a tertiary care hospital in a non-ICU setting can be treated effectively with ampicillin monotherapy without the need for an upgrade to second-and third-line antibiotics. The effectiveness of ampicillin remained high even in children with prehospital antibiotic use. These findings endorse AAP's recommendation and its applicability to resource-limited settings like Nepal.

Second, the majority of the children did not have severe pneumonia and could have been managed using oral antibiotics at primary health centers without the need to visit a tertiary care center like Patan hospital. Therefore, there is a need to create awareness among the public to seek services from primary health centers. Strengthening the primary care will also help to decongest the tertiary care centers. Even in tertiary care settings, children with non-severe CAP may be treated with oral antibiotics.

Third, there were many antibiotic regimens used for treating children, which might be due to the unavailability of written protocol for the treatment of pediatric CAP. Hence, we recommend that a standard treatment protocol for CAP be developed and incorporated into the existing pediatric treatment protocol and implemented urgently. The protocol may also be displayed at several places in the hospital for easy reference. A follow-up study may be conducted to assess changes in practice.

Fourth, the yield of blood culture was extremely low and chest radiographs were normal in the majority of the children. Therefore, we need to reevaluate if these investigations should be a routine practice for all admitted cases of pneumonia, as these can increase the costs of treatment. Chest radiographs may be helpful in detecting the complications and may be considered in severe cases of pneumonia, rather than routinely in all children. 
Finally, the cause of pneumonia could not be established in all the children due to the unavailability of point-of-care, rapid diagnostics. Given the presence of wheezing, low yield of blood culture, and normal chest radiography in the majority of children, we hypothesize that many of the pneumonia cases had viral etiology. This hypothesis is supported by the findings of a previous study from the same hospital [5]. Given the lack of diagnostic capacity in the hospital to confirm viral etiology of pneumonia, the treating pediatricians presumed bacterial etiology and treated every child with antibiotics. Availability of tests like polymerase chain reaction to detect pathogens from respiratory samples like sputum, nasopharyngeal secretions, or biomarkers from blood to differentiate the bacterial or viral etiology can help the pediatricians to decide whether to use antibiotics or not in children with clinically diagnosed pneumonia. This will help in the rational use of antibiotics (including a decision not to use antibiotics in children with viral pneumonia) and the prevention of antimicrobial resistance in the future.

\section{Conclusions}

In conclusion, we found that nearly seven in ten under-five children hospitalized with pneumonia in a tertiary care hospital in Nepal were managed with ampicillin monotherapy with good treatment outcomes. The outcomes remained good even in children with prehospital antibiotic use. Very few children needed second-and third-line antibiotics. This study highlights the effectiveness of ampicillin monotherapy in the treatment of community-acquired pneumonia in hospitalized children in a non-ICU setting.

Author Contributions: Conceptualization and protocol development: B.P., T.K., D.G., B.S.C., A.K. (Ajay Kumar), S.S. (Saw Saw), P.B., S.S. (Shrijana Shrestha) and M.G.; data collection: B.P., P.B., S.K.D., S.G., A.K. (Animesh Khulal) and S.K.Y.; data analysis or interpretation: B.P., T.K., A.K. (Ajay Kumar), B.S.C., S.S. (Saw Saw), D.G., S.S. (Shrijana Shrestha); writing the first draft: B.P., T.K., A.K. (Ajay Kumar), D.G., B.S.C., S.S. (Saw Saw), S.S. (Shrijana Shrestha) and P.B.; critical review of the paper and final approval: B.P., T.K., D.G., A.K. (Ajay Kumar), S.S. (Saw Saw), B.S.C., S.S. (Shrijana Shrestha), M.G., S.K.D., S.G., A.K. (Animesh Khulal), S.G. and S.K.Y. All authors have read and agreed to the published version of the manuscript.

Funding: This SORT IT AMR program was funded by the National Institute of Health Research, Department of Health and Social Care of the United Kingdom, and supported by implementing partners.

Institutional Review Board Statement: Permission to access the data was obtained from the Patan Academy of Health Sciences, Lalitpur Nepal (Ref: drs1911151303). The study was also approved by the Ethics Advisory Group of the International Union against Tuberculosis and Lung Disease, Paris, France (Approval no. 65/19 of 21/08/2019).

Informed Consent Statement: As this was a record review study with no direct interaction with patients, the need for informed patient consent was waived by the ethics committees.

Data Availability Statement: The data that support the findings of this study are available from the corresponding author, B.P., upon reasonable request.

Acknowledgments: This research was conducted through the Structured Operational Research and Training Initiative (SORT IT), a global partnership coordinated by TDR, the Special Programme for Research and Training in Tropical Diseases at the World Health Organization (TDR). The specific SORT IT program that led to these publications included a partnership of TDR with WHO Country offices of Nepal and Myanmar and was implemented along with The Tuberculosis Research and Prevention Center Non-Governmental Organization, Armenia; The International Union Against Tuberculosis and Lung Diseases, Paris and South East Asia offices; Institute of Tropical Medicine, Antwerp, Belgium; Sustainable Health Systems, Freetown, Sierra Leone; Department of Medical Research, Ministry of Health and Sports, Myanmar; School of Public Health, Kathmandu, Nepal; BahirDar University BahirDar, Ethiopia; Centre National de Formation et de Recherche en Santé Rurale de Maferinyah, Guinea; Department of antibiotics and infection control of the public health agency of Sweden; University of Toronto, Canada; and the University of Washington. The authors also thank the Ministry of Health and Population of Nepal, the World Health Organization Country Office 
in Nepal, the Department of Microbiology, and the pediatric research unit under the Department of Pediatrics in the Patan Academy of Health Sciences, who supported us at different stages of the study.

Conflicts of Interest: The authors declare no conflict of interest.

Disclaimer: In any use of this article, there should be no suggestion that WHO endorses any specific organization, products, or services. The views expressed in this article are those of the authors and do not necessarily reflect those of their affiliated institutions. The use of the WHO logo is not permitted. This notice should be preserved along with the article's original URL.

Open Access Statement: In accordance with WHO's open-access publication policy for all work funded by WHO or authored/co-authored by WHO staff members, the WHO retains the copyright of this publication through a Creative Commons Attribution IGO license (http: / / creativecommons. org/licenses/by/3.0/igo/legalcode) which permits unrestricted use, distribution and reproduction in any medium provided the original work is properly cited.

\section{References}

1. Troeger, C.; Blacker, B.; Khalil, I.; Rao, P.C.; Cao, J.; Zimsen, S.R.M.; Albertson, S.B.; Deshpande, A.; Farag, T.; Abebe, Z.; et al. Estimates of the global, regional, and national morbidity, mortality, and aetiologies of lower respiratory infections in 195 countries, 1990-2016: A systematic analysis for the Global Burden of Disease Study 2016. Lancet Infect. Dis. 2018, 18, 1191-1210. [CrossRef]

2. WHO; UNICEF. Global Action Plan for Prevention and Control of Pneumonia (GAPP) Technical Consensus Statement. Bull. World Health Organ. 2009, 86, 322-2A.

3. Rudan, I. Epidemiology and etiology of childhood pneumonia. Bull. World Heal. Organ. 2008, 86, 408-416. [CrossRef] [PubMed]

4. DoHS. Annual Report (2075/76). 2019. Available online: http://dohs.gov.np/dohs-annual-report-fy-2076-77/ (accessed on 29 March 2021).

5. Carter, M.J.; Gurung, P.; Jones, C.; Rajkarnikar, S.; Kandasamy, R.; Gurung, M.; Thorson, S.; Gautam, M.C.; Prajapati, K.G.; Khadka, B.; et al. Assessment of an Antibody-in-Lymphocyte Supernatant Assay for the Etiological Diagnosis of Pneumococcal Pneumonia in Children. Front. Cell. Infect. Microbiol. 2020, 9, 459. [CrossRef]

6. Chhetri, U.D.; Shrestha, S.; Pradhan, R.; Adhikari, N.; Thorson, S.; Pollard, A.J.; Murdoch, D.R.; Kelly, D.F. Clinical Profile of Invasive Pneumococcal Disease in Patan Hospital, Nepal. Kathmandu Univ. Med. J. 2012, 9, 45-49. [CrossRef]

7. WHO. Revised WHO Classification and Treatment of Childhood Pneumonia at Health Facilities: Evidence Summaries; WHO: Geneva, Switzerland, 2014.

8. Chukwuani, C.; Onifade, M.; Sumonu, K. Survey of drug use practices and antibiotic prescribing pattern at a general hospital in Nigeria. Pharm. World Sci. 2002, 24, 188-195. [CrossRef]

9. Hu, S.; Liu, X.; Peng, Y. Assessment of antibiotic prescription in hospitalised patients at a Chinese university hospital. J. Infect. 2003, 46, 161-163. [CrossRef]

10. Orrett, F. Antimicrobial prescribing patterns at a rural hospital in Trinidad: Evidence for intervention measures. Afr. J. Med. Med. Sci. 2001, 30, 161-164.

11. Ayukekbong, J.A.; Ntemgwa, M.; Atabe, A.N. The threat of antimicrobial resistance in developing countries: Causes and control strategies. Antimicrob. Resist. Infect. Control. 2017, 6, 47. [CrossRef]

12. Das, B.; Chaudhuri, S.; Srivastava, R.; Nair, G.B.; Ramamurthy, T. Fostering research into antimicrobial resistance in India. BMJ 2017, 358, j3535. [CrossRef]

13. Plecher, H. Nepal-Statistics \& Facts I Statista. Available online: https://www.statista.com/topics/2427/nepal/ (accessed on 29 March 2021).

14. 10 Facts About Poverty in Nepal I The Borgen Project. Available online: https://borgenproject.org/ten-facts-about-poverty-innepal/ (accessed on 29 March 2021).

15. FMoHP and NHSSP. Budget Analysis of Ministry of Health and Population FY 2018/19 Federal Ministry of Health and Population Policy Planning and Monitoring Division Government of Nepal; Ministry of Health and Population: Kathmandu, Nepal, 2018.

16. Mandell, L.A.; Wunderink, R.G.; Anzueto, A.; Bartlett, J.G.; Campbell, G.D.; Dean, N.C.; Dowell, S.F.; File, T.M.; Musher, D.M.; Niederman, M.S.; et al. Infectious Diseases Society of America/American Thoracic Society Consensus Guidelines on the Management of Community-Acquired Pneumonia in Adults. Clin. Infect. Dis. 2007, 44, S27-S72. [CrossRef]

17. Messinger, A.I.; Kupfer, O.; Hurst, A.; Parker, S. Management of Pediatric Community-acquired Bacterial Pneumonia. Pediatr. Rev. 2017, 38, 394-409. [CrossRef]

18. Dinur-Schejter, Y.; Cohen-Cymberknoh, M.; Tenenbaum, A.; Brooks, R.; Averbuch, D.; Kharasch, S.; Kerem, E. Antibiotic treatment of children with community-acquired pneumonia: Comparison of penicillin or ampicillin versus cefuroxime. Pediatr. Pulmonol. 2012, 48, 52-58. [CrossRef]

19. Nascimento-Carvalho, C.M. Community-acquired pneumonia among children: The latest evidence for an updated management. J. Pediatr. 2020, 96, 29-38. [CrossRef] 
20. Capeding, M.R.; Bravo, L.; Santos, J.; Kilgore, P.E.; Kim, S.A.; Balter, I.; Hubler, R.; Ye, J.; Moscariello, M. Prospective Surveillance Study of Invasive Pneumococcal Disease Among Urban Children in the Philippines. Pediatr. Infect. Dis. J. 2013, 32, e383-e389. [CrossRef]

21. Nguyen, P.T.; Tran, H.T.; Fitzgerald, D.A.; Tran, T.S.; Graham, S.M.; Marais, B.J. Characterisation of children hospitalised with pneumonia in central Vietnam: A prospective study. Eur. Respir. J. 2019, 54, 1802256. [CrossRef]

22. Jullien, S.; Pradhan, D.; Tshering, T.; Sharma, R.; Dema, K.; Garcia-Garcia, S.; Ribó, J.L.; Muñoz-Almagro, C.; Bassat, Q. Pneumonia in children admitted to the national referral hospital in Bhutan: A prospective cohort study. Int. J. Infect. Dis. 2020, 95, 74-83. [CrossRef]

23. Youssef, A.S.; Fanous, M.; Siddiqui, F.J.; Estrada, J.; Chorny, V.; Braiman, M.; Mayer, E.F. Value of Blood Cultures in the Management of Children Hospitalized with Community-Acquired Pneumonia. Cureus 2020, 12, e8222. [CrossRef]

24. Neuman, M.I.; Hall, M.; Lipsett, S.C.; Hersh, A.L.; Williams, D.J.; Gerber, J.S.; Brogan, T.V.; Blaschke, A.J.; Grijalva, C.G.; Parikh, K.; et al. Utility of Blood Culture Among Children Hospitalized With Community-Acquired Pneumonia. Pediatrics 2017, 140, e20171013. [CrossRef]

25. Jain, S.; Williams, D.J.; Arnold, S.R.; Ampofo, K.; Bramley, A.M.; Reed, C.; Stockmann, C.; Anderson, E.J.; Grijalva, C.G.; Self, W.H.; et al. Community-Acquired Pneumonia Requiring Hospitalization among U.S. Children. N. Engl. J. Med. 2015, 372, 835-845. [CrossRef] [PubMed]

26. Byington, C.; Bradley, J. Pediatric Community-Acquired Pneumonia. In Feigin and Cherry's Textbook of Pediatric Infectious Diseases; Elsevier Saunders: Philadelphia, PA, USA, 2014.

27. Tam, P.-Y.I.; Bernstein, E.; Ma, X.; Ferrieri, P. Blood Culture in Evaluation of Pediatric Community-Acquired Pneumonia: A Systematic Review and Meta-analysis. Hosp. Pediatr. 2015, 5, 324-336. [CrossRef]

28. Lipsett, S.C.; Hall, M.; Ambroggio, L.; Desai, S.; Shah, S.S.; Brogan, T.V.; Hersh, A.L.; Williams, D.J.; Grijalva, C.G.; Gerber, J.S.; et al. Predictors of Bacteremia in Children Hospitalized with Community-Acquired Pneumonia. Hosp. Pediatr. 2019, 9, 770-778. [CrossRef]

29. Hazir, T.; Bin Nisar, Y.; Qazi, S.; Khan, S.F.; Raza, M.; Zameer, S.; Masood, S.A. Chest radiography in children aged 2-59 months diagnosed with non-severe pneumonia as defined by World Health Organization: Descriptive multicentre study in Pakistan. BMJ 2006, 333, 629. [CrossRef] [PubMed]

30. Hassen, M.; Toma, A.; Tesfay, M.; Degafu, E.; Bekele, S.; Ayalew, F.; Gedefaw, A.; Tadesse, B.T. Radiologic Diagnosis and Hospitalization among Children with Severe Community Acquired Pneumonia: A Prospective Cohort Study. BioMed Res. Int. 2019, 2019, 6202405. [CrossRef]

31. Harris, M.; Clark, J.; Coote, N.; Fletcher, P.; Harnden, A.; Mckean, M.; Thomson, A.; on behalf of the British Thoracic Society Standards of Care Committee. British Thoracic Society guidelines for the management of community acquired pneumonia in children: Update 2011. Thorax 2011, 66 (Suppl. 2), ii1-ii23. [CrossRef] [PubMed]

32. Mahabee-Gittens, E.M.; Grupp-Phelan, J.; Brody, A.S.; Donnelly, L.F.; Bracey, S.E.A.; Duma, E.M.; Mallory, M.L.; Slap, G.B. Identifying Children with Pneumonia in the Emergency Department. Clin. Pediatr. 2005, 44, 427-435. [CrossRef]

33. Lipsett, S.C.; Monuteaux, M.C.; Bachur, R.G.; Finn, N.; Neuman, M.I. Negative Chest Radiography and Risk of Pneumonia. Pediatrics 2018, 142, e20180236. [CrossRef] 